Florida State University College of Law

Scholarship Repository

Scholarly Publications

$1-2012$

\title{
Economics of the Independent Invention Defense under Incomplete Information
}

Murat C. Mungan

Florida State University College of Law

Follow this and additional works at: https://ir.law.fsu.edu/articles

Part of the Intellectual Property Law Commons, and the Law and Economics Commons

\section{Recommended Citation}

20 Sup Ct Econ Rev 183-203 (2012)

This Article is brought to you for free and open access by Scholarship Repository. It has been accepted for inclusion in Scholarly Publications by an authorized administrator of Scholarship Repository. For more information, please contact efarrell@law.fsu.edu. 


\section{CHICAGO JOURNALS}

Economics of the Independent Invention Defense under Incomplete Information Author(s): Murat C. Mungan

Source: Supreme Court Economic Review, Vol. 20, No. 1 (January 2012), pp. 183-203

Published by: The University of Chicago Press

Stable URL: http://www.jstor.org/stable/10.1086/668519

Accessed: 28/01/2015 12:12

Your use of the JSTOR archive indicates your acceptance of the Terms \& Conditions of Use, available at http://www.jstor.org/page/info/about/policies/terms.jsp

JSTOR is a not-for-profit service that helps scholars, researchers, and students discover, use, and build upon a wide range of content in a trusted digital archive. We use information technology and tools to increase productivity and facilitate new forms of scholarship. For more information about JSTOR, please contact support@ jstor.org. 


\section{Economics of the Independent Invention Defense under Incomplete Information}

Murat C. Mungan*

Patents lead to ex post deadweight loss arising from a noncompetitive market structure for the invention. Many have argued that introducing independent invention as a defense (IID) to patent infringement can increase social welfare by decreasing such deadweight loss at the price of a modest decrease in the number of inventions. This paper considers the effects of IID in a setting where R $\Theta D$ firms have incomplete information about their rivals. Four main results follow under incomplete information: (i) fewer things are invented under an IID regime; (ii) IID's effects on welfare are ambiguous; (iii) IID is more likely to increase welfare if gains from competition in the product market are high; and (iv) determining precise conditions under which IID performs better than the current regime requires access to data that are extremely hard to find and quantify.

\section{IN T R O D U C T I O N}

Patent protections enable potential inventors to benefit from their investments. Absent protections, inventors' incentives would be undesirably low and retard technological progress. On the other hand,

* Visiting Assistant Professor of Law, Florida State University, College of Law, 425 W. Jefferson Street, Tallahassee, FL 32306. e-mail: mmungan@law.fsu.edu. I would like to thank Samson Vermont, the editor, and an anonymous referee for useful comments and suggestions. The usual disclaimer applies.

(C) 2012 by the University of Chicago. All rights reserved. 978-0-226-76764-2/2012/0020-0005\$10.00 
rights conferred on inventors through patent law generate ex post costs arising from noncompetitive market dynamics. This usecreation trade-off is the focal point of many articles discussing potential patent law reforms. A new and expanding branch of literature discusses the possibility of reforming patent law by making independent invention a defense to patent infringement. ${ }^{1}$

An independent invention defense (IID) may take many forms. For purposes of this paper, it refers to any situation where an independent inventor is allowed to use, sell or make the invention. In what follows, IID may refer to a legal regime where independent invention is allowed as a defense or the defense itself. ${ }^{2}$ I will call a legal regime which does not allow IID the current regime, and call the invention the product.

Those who advocate IID rely on the fact that it would reduce ex post deadweight losses. ${ }^{3}$ Most proponents of IID acknowledge the fact that the defense would likely suppress creation incentives but argue that this does not offset benefits from reducing deadweight losses. ${ }^{4}$ In this paper I investigate under what conditions IID proponents' claims hold by modeling the interactions between two R\&D firms under IID and the current regime, and under complete and incomplete information settings.

In Section II, I consider a very simple complete information framework. Two R\&D firms simultaneously choose whether or not to conduct research to invent a particular product. Firms that conduct re-

\footnotetext{
${ }^{1}$ For a discussion of the independent invention defense and related issues, see $\mathrm{P}$. Dasgupta and J. Stiglitz, Uncertainty, Industrial Structure, and the Speed of $R \uplus D, 11$ Bell J of Econ 1 (1980); M. La Manna, R. Macleod, and D. de Meza, The Case of Permissive Patents, 33 European Econ Rev 1427 (1989); S.M. Maurer and S. Scotchmer, Independent Invention Defence in Intellectual Property, 69 Economica 535 (2002); V. Denicolo and L.A. Franzoni, Patents, Secrets, and the First-Inventor Defense, 13 J Econ \& Mgmt Strategy 517 (2004); C. Shapiro, Prior User Rights, 96 AEA Papers \& Proceed 92 (2006); C. Shapiro, Patent Reform: Aligning Reward and Contribution, 8 Innovation Policy ↔) Econ 111 (2007); M.A. Lemley, Should Patent Infringement Require Proof of Copying?, 105 Mich L Rev 1525 (2007); E. Henry, Runner-up Patents: Is Monopoly Inevitable?, 112 Scandinavian J Econ 417 (2010).

${ }^{2}$ I will sometimes refer to the regime as the IID regime.

${ }^{3}$ I refer to costs arising from noncompetitive market dynamics, collectively, as deadweight losses. These can be traditional deadweight losses arising from monopoly pricing, or others arising from the patentee's reluctance to license its patent (as in Maurer and Scotchmer, 69 Economica 535 (cited in note 1)). There is another, ex ante, cost considered in this paper and in id, namely duplication of research costs. However, deadweight losses are the main concern of this and other papers in the literature, since their magnitude is likely to be greater.

${ }^{4}$ Maurer and Scotchmer, 69 Economica 535 (cited in note 1) can be classified as arguing for weaker patents, but my paper abstracts from weaker patents' negative effect on the level of innovation. This is because Maurer and Scotchmer assume that $\mathrm{R} \& \mathrm{D}$ investment is lump-sum and results in certain invention. Id.
} 
search invent the product with an interior probability. I capture the interactions between firms using a simple normal form game. Legal regimes (that is, IID and the current legal regime) are incorporated through their effects on firms' rights in cases where both firms invent the product. By comparing equilibria under the two legal regimes, I identify conditions under which IID results in greater social welfare than does the current regime.

In Section III, I extend the analysis to incomplete information. The existence of different types of potential inventors is captured conveniently by assigning them different likelihoods of success. I incorporate this heterogeneity by considering a static Bayesian game. In this framework, R\&D firms draw their probability of success from a known distribution of probabilities. Each firm acquires knowledge of its own probability of success, but not its rival's. As with complete information, legal regimes affect equilibria by providing different rights in cases where both firms invent the product. I identify conditions under which IID is superior to the current regime.

In Section IV, I compare results obtained under the complete and incomplete information settings. Then I suggest a few topics for future research and discuss the implications of the incomplete information framework. The last portion of this section concludes. Mathematical proofs to all propositions found in Section III are contained in the Appendix.

\section{COMPLETE INFORMATION}

Consider two firms, which may incur research and development expenses of $C$ in order to invent a new product with a probability of $p$. They make this decision simultaneously. The invention produces welfare $W_{m}$ under the current regime and welfare $W_{d}$ under IID. ${ }^{5}$ Under current law, if both firms are successful in inventing the product, they are equally likely to be rewarded with a patent that grants payoff $\pi_{m}$. A firm that fails to obtain a patent earns zero profit. In IID, on the other hand, if both firms are successful, both are entitled to market profits $\pi_{d \cdot}{ }^{6}$ I assume that $(1 / 2) \pi_{m}>\pi_{d}>C .^{7}$

${ }^{5} W_{m}$ and $W_{d}$ include firms' profits.

${ }^{6}$ Conclusions would not change if the first and second firms were entitled to $\pi_{1}>\pi_{2}$, respectively. In this case, $\pi_{d}$ would be the expected profits of a firm, which would be obtained by calculating a weighted average of $\pi_{1}$ and $\pi_{2}$.

${ }^{7}$ The first inequality would follow in any reasonable model. If $(1 / 2) \pi_{m}<\pi_{d}$, then the single patent holder under current law can improve his profits by creating a subsidiary and giving him rights that would be conferred to an independent inventor in an IID. 


\section{A. Nash Equilibria under Current Law}

Since decisions to engage in $R \& D$ are made simultaneously, interactions among firms are best described by a simple normal form game. Let $R$ denote a firm's strategy to engage in research and $\underline{R}$ to refrain. Under current law, firms face the following game:

Table 1. Normal Form Representation under Current Law

\begin{tabular}{lll}
\hline Player 1/2 & \multicolumn{1}{c}{$R$ (Research) } & $\underline{R}$ (No Research) \\
\hline$R$ (Research) & $\left\{\left(p-\left(p^{2} / 2\right)\right) \pi_{m}-C\right\},\left\{\left(p-\left(p^{2} / 2\right)\right) \pi_{m}-C\right\}$ & $\left\{p \pi_{m}-C\right\},\{0\}$ \\
$\underline{R}$ (No Research) & $\{0\},\left\{p \pi_{m}-C\right\}$ & $\{0\},\{0\}$ \\
\hline
\end{tabular}

The pure Nash Equilibria of this game depend on the value of $p$. In particular the strategy profile $\{R, R\}$ is the unique Nash Equilibrium iff

$$
\left(p-\left(p^{2} / 2\right)\right) \pi_{m}>C
$$

Inequality (1) holds if $p$ is above a critical value, since the left-hand side of the inequality is increasing in $p$ and attains its maximum at $(1 / 2) \pi_{m}$, which is greater than $C$. This critical value is given by:

$$
\underline{p} \equiv 1-\sqrt{1-2 \frac{C}{\pi_{m}}}
$$

For intermediate values of $p$, equilibrium will be achieved where only a single firm engages in research. If, on the other hand, $p$ is low, neither firm will conduct research. In other words, $\{R, \underline{R}\}$ and $\{\underline{R}, R\}$ will be equilibria if $\left(C / \pi_{m}\right)<p<\underline{p}$, and $\{\underline{R}, \underline{R}\}$ will be the equilibrium if $p<\left(C / \pi_{m}\right)$. These equilibria can be summarized as follows: ${ }^{8}$

Table 2. Equilibria under Current Law

\begin{tabular}{ll}
\hline Equilibria & Condition \\
\hline$\{R, R\}$ & if $p \geq \underline{p}$ \\
$\{\underline{R}, R\}$ and $\{R, \underline{R}\}$ & if $\underline{p}>p \geq\left(C / \pi_{m}\right)$ \\
$\{\underline{R}, \underline{R}\}$ & if $\left.\mid C / \pi_{m}\right)>p$ \\
\hline
\end{tabular}

${ }^{8}$ If $p=\underline{p}$, the game has three Nash Equilibria, namely, all profiles except $\{\underline{R}, \underline{R}\}$. Similarly, when $p=\left(C / \pi_{m}\right)$, all profiles except $\{R, R\}$ are equilibria. In these exceptional cases I assume that parties will end up in an equilibrium involving the most research. 


\section{B. Nash Equilibria under IID}

Table 1 can easily be manipulated to represent firms' interactions under the IID regime:

Table 3. Normal Form Representation under IID

\begin{tabular}{lll}
\hline Player $1 / 2$ & \multicolumn{1}{c}{$R$ (Research) } & $\underline{R}$ (No Research) \\
\hline$R$ (Research) & $\left\{p^{2} \pi_{d}+p(1-p) \pi_{m}-C\right\},\left\{p^{2} \pi_{d}+p(1-p) \pi_{m}-C\right\}$ & $\left\{p \pi_{m}-C\right\},\{0\}$ \\
$\underline{R}$ (No Research) & $\{0\},\left\{p \pi_{m}-C\right\}$ & $\{0\},\{0\}$ \\
\hline
\end{tabular}

The analysis to determine Nash Equilibria under IID is very similar to that of the current regime. The only thing that changes is the critical value of $p$ that determines the condition under which $\{R, R\}$ is the unique pure strategy equilibrium of the game. This critical value, $p$, is implicitly defined by:

$$
p^{2} \pi_{d}+p(1-p) \pi_{m}=C
$$

To verify the existence and uniqueness of $p$, let $z(p) \equiv p^{2} \pi_{d}+$ $p(1-p) \pi_{m}$. It follows that $z$ is concave, $z(0)=0$, and $z(1)=\pi_{d}$. This implies that there exists a unique and interior $p$ such that $0<z(p)=$ $C<\pi_{d^{\prime}}$ and $z(p)>C$ for all $p \in(p, 1]$. The equilibria under IID can be summarized by replacing $\underline{p}$ with $p$ in Table 2 :

Table 4. Equilibria under IID

\begin{tabular}{ll}
\hline Equilibria & Condition \\
\hline$\{R, R\}$ & if $p \geq p$ \\
$\{\underline{R}, R\}$ and $\{R, \underline{R}\}$ & if $p>p \geq\left(C / \pi_{m}\right)$ \\
$\{\underline{R}, \underline{R}\}$ & if $\quad\left(C / \pi_{m}\right)>p$ \\
\hline
\end{tabular}

\section{Comparing Regimes}

To compare regimes, it will be useful to identify the relation between $p$ and $p$. Note that $z$ is increasing for all $p<p$. This observation, coupled with the fact that $z(p)$ is smaller than the left hand side of inequality ( 1 ) for all positive $p$, implies that $p>\underline{p}$. Having determined the relationship between $\underline{p}$ and $p$, Tables 2 and 4 can be combined to compare the effects of legal regimes: 
Table 5. Comparison of Equilibria

\begin{tabular}{lll}
\hline $\begin{array}{l}\text { Equilibria under } \\
\text { IID }\end{array}$ & $\begin{array}{l}\text { Equilibria under } \\
\text { Current Regime }\end{array}$ & Condition \\
\hline$\{R, R\}$ & $\{R, R\}$ & if $p \geq p$ \\
$\{R, R\}$ and $\{R, \underline{R}\}$ & $\{R, R\}$ & if $p>p \geq \underline{p}$ \\
$\{\underline{R}, R\}$ and $\{R, \underline{R}\}$ & $\{\underline{R}, R\}$ and $\{R, \underline{R}\}$ & if $\underline{p}>p \geq\left(C / \pi_{m}\right)$ \\
$\{\underline{R}, \underline{R}\}$ & $\{\underline{R}, \underline{R}\}$ & if $\left(C / \pi_{m}\right)>p$
\end{tabular}

Table 5 shows that equilibria under the two legal regimes differ from each other only when $p$ is intermediate (that is, $p \in[p, p)$ ). Here, both firms conduct research under the current regime, but a single firm conducts research under IID. This result follows from the fact that firms prefer monopoly profits half the time over duopoly profits all the time (that is, $\left.\left(\pi_{m} / 2\right)>\pi_{d}\right)$. Since $\left(\pi_{m} / 2\right)>\pi_{d}$ the current regime leads to greater expected payoffs in cases where both firms conduct research. Therefore, the current regime incentivizes both firms to conduct research in cases where $p$ is only intermediate, whereas only one firm conducts research under IID. This result is summarized by the following observation. ${ }^{9}$

Observation 1: Research by multiple firms is observed less frequently under IID, implying that fewer things are invented.

This observation alone, however, does not let us draw conclusions concerning IID's welfare effects. While IID reduces the number of inventions it also leads to (i) lower research costs and (ii) higher surplus in the product market when both firms invent the product. Whether gains from an IID regime offset its costs will depend on a number of conditions, which are explored next.

In evaluating the desirability of IID, define social welfare as the sum of firms' profits and consumer surplus $\left(W_{m}\right.$ or $\left.W_{d}\right)$ minus costs incurred for research $(C)$. Let $V_{N}$ and $V_{D}$ denote social welfare under the current regime and IID, respectively. To identify IID's welfare effects it is useful to categorize the probability of success, $p$, into four categories: (I) high $(p>p)$, (II) intermediate $(p>p>\underline{p})$, III) low $\left(\underline{p}>p \geq\left(\mathrm{C} / \pi_{\mathrm{m}}\right)\right)$, and IV) very low $\left(\left(C / \pi_{\mathrm{m}}\right)>p\right)$.

\footnotetext{
${ }^{9}$ Henry, Runner-up Patents, 112 Scandinavian J Econ 417 (cited in note 1), in Proposition 1, and Maurer and Scotchmer, 69 Economica 535 (cited in note 1), in Proposition 2, derive results similar to Observation 1.
} 


\section{High $p$}

When $p$ is high both firms conduct research in both regimes. However, social welfare is higher under IID because of reduced deadweight loss in the product market. The expected levels of social welfare are given by:

$$
\begin{aligned}
& V_{N}=\left(p^{2}+2 p(1-p)\right) W_{m}-2 C \text { and } \\
& V_{D}=p^{2} W_{d}+2 p(1-p) W_{m}-2 C
\end{aligned}
$$

This implies that $V_{D}>V_{N}$ because $W_{d}>W_{m}$.

\section{Intermediate $p$}

Under current law, Tables 2 and 4 imply that multiple firms engage in research when $p$ is intermediate. However, under IID only one firm conducts research. In this case, the desirability of IID depends on several factors, which can be identified by comparing welfare under IID with the current regime:

$$
V_{N}=\left(p^{2}+2 p(1-p)\right) W_{m}-2 C \text { and } V_{D}=p W_{m}-C
$$

Therefore, $V_{N}>V_{D}$ if

$$
p(1-p) W_{m}>C
$$

Note that this condition may or may not be satisfied by intermediate $p^{\prime}$ s. To verify this claim consider two economies ( 1 and 2$)$ where economy $i$ is described by the list $E_{i}=\left\langle C_{i}, \pi_{m, i} W_{m, i}\right\rangle$. Other values that may distinguish economies are not included in the list because they do not affect the argument. Let

$$
E_{1}=<3,8,11>\text { and } E_{2}=<3,8,40>
$$

Plugging in relevant values in $(2)$ reveals that $\underline{p}=(1 / 2)$ in both economies, which by definition is an intermediate probability. Hence, (6) holds at $\underline{p}$ iff

$$
1 / 4>C / W_{m}
$$

which is true for $E_{2}$ but not for $E_{1}$. Next, note that in $E_{1}$, no intermediate probability satisfies $(6)$, since $1 / 4>p(1-p)$ for all $p$. Finally, note that $W_{m}$ does not have an upper bound. Therefore, for any range of intermediate probabilities, one can create an economy similar to 
$E_{2}$, in which (6) is satisfied for all intermediate probabilities. In other words, the desirability of IID is ambiguous for intermediate probabilities. This example also illustrates the importance of consumer surplus $\left(W_{m}-\pi_{m}\right)$ in determining the desirability of IID.

\section{Low and Very Low $p$ 's}

The analysis of these two cases is rather straightforward and leads to the intuitive result that regimes do not affect welfare. When $p$ is low, only a single firm conducts research under both regimes. Hence, $V_{N}=V_{D}=p W_{m}-C$. When $p$ is very low, neither firm conducts research, hence $V_{N}=V_{D}=0$.

\section{Summary of Results under Complete Information}

The simple complete framework captures many intuitive results. These can be summarized as follows. ${ }^{10}$

Proposition 1: Under complete information:

(i) fewer things are invented in an IID regime;

(ii) IID generates higher welfare for products that are likely to be invented once invested in (high p);

(iii) for products with an intermediate likelihood of being invented (intermediate $p$ ), welfare effects are ambiguous and IID is likely to dominate if the cost of research $(C)$ is close to the social value of the invention $\left(W_{m}\right)$; and

(iv) for products with a low likelihood of being invented the current regime and IID produce the same results.

Proof: Follows immediately from the discussion in Section IIC.

Proposition 1 suggests that IID is likely to dominate the current regime (weakly) if research costs are close to the social value of the product. When this condition does not hold, IID is likely to dominate the current regime when applied to inventions that have a high likelihood of being invented.

${ }^{10}$ Existing literature comes to conclusions similar to those summarized by Proposition 1. See, for example, note 9. See also Henry, 112 Scandinavian J Econ 417 (cited in note 1), Proposition 2; and C. Shapiro, Prior User Rights, 96 AEA Papers \& Proceed 92 (2006), Theorem 1, acknowledging the ambiguity of the desirability of runner-up patents and prior user rights, respectively, and deriving parametric conditions under which they are desirable. 


\section{INCOMPLETE INFORMATION}

In this section, I construct a model in which two firms randomly draw their probabilities of inventing a product from a known distribution. After this draw, a firm learns its own probability of inventing the product but not its competitor's. The first and second firms' probabilities of success are denoted by $p_{1}$ and $p_{2^{\prime}}$ respectively. Unlike in the complete information case, $p_{i \in\{1,2\}}$ are assumed to be random variables independently and identically distributed with density function $f(.) .{ }^{11}$ Once $p_{i \in\{1,2\}}$ are realized, each firm decides whether or not to conduct research. If firm $i$ conducts research, it incurs a cost of $C$ and succeeds with probability $p_{i}$ otherwise it exits the game. ${ }^{12}$

Slightly manipulating Tables 1 and 3 enables us to identify firms' expected payoffs given $p_{i \in\{1,2\}}$ and their decisions concerning research:

Table 6. Expected Payoffs under Incomplete Information

\begin{tabular}{lll}
\hline \multicolumn{1}{c}{$R$ Player $1 / 2$} & $\underline{R}$ (Nesearch) & \\
\hline$R($ Research $)$ & $\left\{p_{1}\left(1-p_{2}\right) \pi_{m}+p_{1} p_{2} \rho-C\right\},\left\{p_{2}\left(1-p_{1}\right) \pi_{m}+p_{1} p_{2} \rho-C\right\}$ & $\left\{p_{1} \pi_{m}-C\right\},\{0\}$ \\
$\underline{R}$ (No Research) $)\{0\},\left\{p_{2} \pi_{m}-C\right\}$ & $\{0\},\{0\}$ \\
\hline
\end{tabular}

${ }^{11}$ Assuming lack of knowledge concerning the probability of success is only one way of incorporating incomplete information. This approach is a simple and intuitive way of capturing heterogeneities across firms in a single dimension. Heterogeneities across any relevant characteristic(s) would ultimately reflect a firm's relative "advantage" or "disadvantage." Accordingly, alternative specifications, for example where firms' cost of conducting research differ, are likely to produce similar results. Collecting heterogeneities in a single dimension is not a novel idea; it has been used repeatedly in the incomplete information framework. See, for example, D.R. Deere, Bilateral Trading as an Efficient Auction over Time, 96 J Pol Econ 100(1988) (capturing privately observable heterogeneities across firms through their productivity levels); D.P. Baron and R.B. Myerson, Regulating a Monopolist with Unknown Costs, 50 Econometrica 911 (1982) (modeling heterogeneities across firms through a single cost parameter).

${ }_{12}$ An alternative model could consider probabilities of success, which depend on (i) investments made by the firm and (ii) an exogenous productivity parameter drawn from a known distribution. In this case, the analysis could be conducted in a very similar way by treating the productivity parameter as only privately observable. Such a model would produce qualitatively similar results, because the productivity parameter would collect all heterogeneity across firms and would reflect whether a particular firm has made a relatively good or bad draw. Quantitative results, on the other hand, would necessarily depend on the specific functional relationship between a firm's probability of success, investments, and productivity parameter. 
where

$$
\rho(L)=\left\{\begin{array}{lll}
\pi_{d} & \text { if } & L=D \\
\pi_{m / 2} & \text { if } & L=N
\end{array}\right.
$$

denotes profits when both inventors are successful as a function of the legal regime chosen, and $L \in\{D, N\}$ describes the legal regime where $D$ is IID and $N$ is the current regime.

Since neither firm knows the other firm's probability of success, Table 6 cannot be used to determine the equilibrium. This lack of knowledge forces firms to base the decision whether or not to conduct research only on their own probability of success. To formalize this observation, consider the standard static Bayesian framework ${ }^{13}$ and let $a_{i} \in A=\{R, \underline{R}\}$ denote firm $i^{\prime}$ s choice to conduct research or to refrain. Next, let $s_{i}():.[0,1] \rightarrow A$ denote firm $i^{\prime}$ s strategy to conduct research based on its realized probability of success. Define $u_{i}$ as firm i's expected payoff, which depends on its rival's and its own choice of conducting research, and its realized probability of success. That is $u_{i}=u_{i}\left(a_{i}, a_{i} ; p_{i}\right)$, where $j$ is the index for $i^{\prime}$ s rival. The values $u_{i}$ takes, given the legal regime, can easily be determined using Table 6:

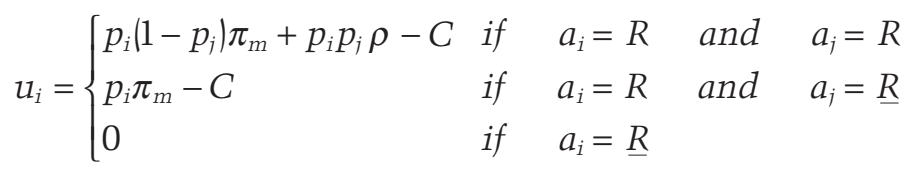

\section{A. Equilibrium Properties}

Given this notation, each firm seeks to maximize its expected profits by choosing an action from $A$ for every possible probability of success. The equilibrium consists of a strategy profile $S^{*}=\left(S_{1}{ }^{*}, S_{2}{ }^{*}\right)$ where $s_{i}{ }^{*}$ solves:

$$
\max _{a_{i \in A}} \int_{0}^{1} u_{i}\left(s_{j}^{*}\left(p_{j}\right), a_{i} ; p_{i}\right) \mathrm{f}\left(p_{j}\right) d_{p j}
$$

for all $p_{i}$, and for all $i \in\{1,2\}$.

To characterize the solution of this game, note that a firm's payoff, given the strategy of its potential rival, increases in its probability of success regardless of the legal regime chosen. Accordingly, if

13 The reader unfamiliar with static Bayesian games may consult R. Gibbons, Game Theory for Applied Economists, ch 3 (Princeton 1992). I use similar notation to Gibbons', where $s_{i}$ and $a_{i}$ represent, respectively, the strategy and action chosen by player $i$. In Gibbons' framework, $p_{i}$ would be called player $i^{\prime}$ s type and denoted by $t_{i}$. 
a firm is prepared to continue research after drawing a probability of success $p_{1}$ it will certainly continue research if it draws a higher probability $p_{h}>p_{l}$. A corollary of this observation is that any strategy that is a best response (to any other strategy) must be one where the firm continues research if and only if it draws a probability that is higher than some critical likelihood of success. This intuitive conjecture is verified and formalized by the following proposition:

Proposition 2: Under both legal regimes, (i) given any strategy chosen by firm i, firm i's expected payoff from conducting research is increasing in $p_{i}$, and (ii) accordingly, any equilibrium $S^{*}=\left(S_{1}{ }^{*}, S_{2}{ }^{*}\right)$ must consist of strategies of the form ${ }^{14}$

$$
S_{i}^{*}\left(p_{i}\right)= \begin{cases}R & \text { if } p_{i} \geq p_{i}^{C} \\ \underline{R} & \text { otherwise }\end{cases}
$$

\section{Proof: See Appendix.}

The intuition behind this result is best described by interpreting a high $p$ draw as one that puts that firm in a relatively advantageous state: $f(p)$ captures firms' beliefs concerning their rivals' unobservable likelihood of inventing the product..$^{15} \mathrm{~A}$ firm will guess that its rival will (not) conduct research if its rival has good (bad) characteristics. Therefore, given $f(p)$ a firm will have a belief concerning how frequently its rival will conduct research. By using this estimate a firm can calculate how high its probability of success $p_{i}^{C}$ must be for it to have positive expected payoffs from conducting research.

Absent further restrictions, there are infinitely many equilibria that satisfy Proposition 2. This follows from the fact that there can be asymmetric equilibria where the threshold probability for firm 1 $\left(p_{1}{ }^{C}\right)$ is close to zero, but the same for firm $2\left(p_{2}{ }^{C}\right)$ is close to 1 . This possibility not only creates unrealistic results but is hard to interpret. To avoid these problems, I focus on symmetric equilibria. This focus will allow me to compare two unique symmetric Bayesian Nash Equilibria, one where independent invention is allowed as a defense and one where it is not. I start by identifying the unique symmetric equilibria under both regimes using the following definitions.

${ }^{14}$ I assume that indifferent firms always choose to continue research.

${ }^{15}$ An implicit assumption is that firms do not reevaluate their beliefs concerning their rival's type after learning their own types. See Section IV.C, discussing this assumption. 
Definition 1: A strategy of the form

$s_{i}\left(p_{i}\right)= \begin{cases}R & \text { if } p_{i} \geq p^{C} \\ \underline{R} & \text { otherwise }\end{cases}$

will be denoted as $s_{p}^{c}$, and any symmetric strategy profile $\left(s_{p}^{c}, s_{p}^{c}\right)$, will be denoted as $S_{p}^{c}$.

Before comparing the properties of symmetric equilibria under different legal regimes, it is worth formalizing the fact that in each regime there exists a unique symmetric equilibrium $\mathrm{S}_{p}{ }^{c}$, and that $p^{C}$ is interior:

Proposition 3: In each legal regime, there is a unique symmetric Pure Strategy Bayesian Nash Equilibrium $\mathrm{S}_{p}{ }^{c}$, where $p^{C}$ $\in(0,1)$.

Proof: See Appendix.

\section{B. Comparing Regimes}

Having established the existence and uniqueness of symmetric equilibria under each regime, one can compare the properties of these two equilibria. Part (ii) of Proposition 2 and Definition 1 suggest that symmetric equilibria can be characterized by a single parameter, $p^{C}$; that is, the threshold probability that determines whether or not firms conduct research. The difference between these two probabilities under the two legal regimes will be crucial in identifying benefits and costs that come with these regimes. The next proposition establishes a general relationship between these probabilities.

Proposition 4: Let the symmetric equilibrium under the current legal regime and IID be denoted $S_{p}{ }^{N}$ and $S_{p}{ }^{D}$, respectively. Then it follows that $p^{N}<p^{D}$.

Proof: See Appendix.

An IID regime increases the threshold probability of success by reducing expected profits from inventing the product. This result implies that an IID regime leads to less research:

Corollary: Fewer things are invented in an IID regime.

However, fewer inventions do not necessarily mean less welfare. One benefit of IID is savings from research costs. A second obvious advantage of IID is less deadweight loss in the product market. In general neither regime dominates the other, since welfare effects depend on the particular draw $\left(p_{1}, p_{2}\right)$. One can, however, 


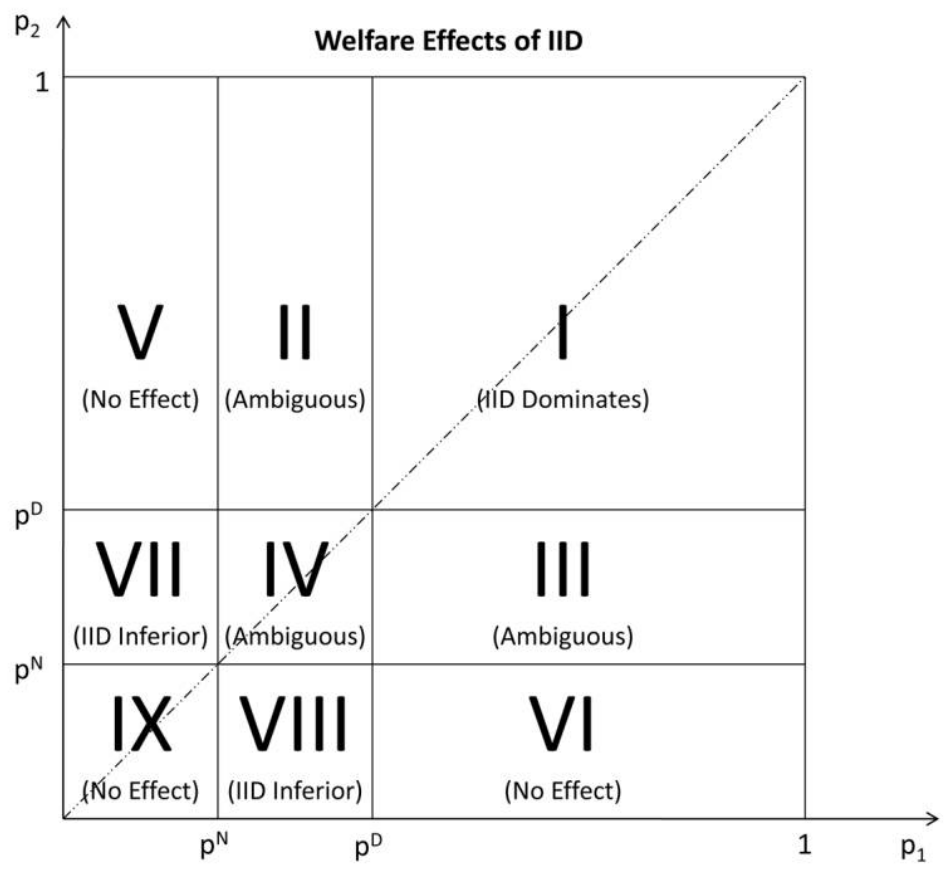

Figure 1. Welfare effects of IID

identify particular regions in $p_{1}-p_{2}$ space that imply welfare gains (losses) from an IID regime. Figure 1 partitions the $p_{1}-p_{2}$ space into nine areas according to the incentives provided to competing firms.

In determining the significance and meaning of areas I-IX, it is useful to identify welfare effects associated with these areas:

Proposition 5: In an IID regime, social welfare is (i) higher in area I, (ii) lower in areas VII and VIII, and (iii) the same in areas V,VI, and IX. (iv) Absent further assumptions, welfare effects are ambiguous in areas II, III, and IV.

Proof: See Appendix.

In area I, both firms conduct research under each regime. Hence, research costs are equal. If only a single firm invents the product, a monopoly emerges so that social benefits associated with the invention are equal in both regimes. However, when both firms invent the product, the IID regime obviously produces higher welfare, because of increased competition in the product market. Hence, IID produces higher expected social welfare in area I than the current regime. 
In areas V and VI, a single firm conducts research under either regime. The result is a monopoly and the same social welfare in both cases. In area IX neither firm conducts research in either regime. The result is zero welfare in both cases.

In areas VII and VIII neither firm conducts research under an IID regime. However, one firm conducts research under the current regime. Under the current regime, by definition, firms engaging in research are doing so because they expect profits that exceed the cost of research. This implies that research is desirable, since social benefits from the invention are at least as great as the firm's private profits that exceed the cost of research. Hence, IID leads to less welfare than the current regime in these areas, because it eliminates desirable research.

In areas II and III, expected profits under IID are only high enough to incentivize a single firm, whereas the current regime leads to research by both firms. In the current regime, whether marginal benefits from the second firm's research associated with a higher likelihood of invention are great enough to warrant their (research) costs is ambiguous. But when the first firm's probability draw is close to 1 , the second firm's research efforts are certainly wasteful. This follows because the second firm incurs research costs, only to affect the probability of invention slightly, and without increasing competition after the product is invented. Hence, areas II and III contain regions in which IID improves welfare. Moreover, IID results in higher welfare in the entirety of areas II and III when the difference between the invention's social benefit and research costs is small. A sufficient condition for IID to dominate in these areas is provided and formalized in Proposition 6.

In area IV, neither firm conducts research in an IID regime, while both firms conduct research in the current regime. Absent further assumptions it is unclear whether the increased probability of invention due to the second firm's research is worth its research costs. In general, IID is more likely to be desirable when the value of the invention is close to costs of research. This is formalized by the following proposition.

Proposition 6: (i) Social welfare is higher under an IID regime in areas II and III if $W_{m}<4 C$, and (ii) there always exists $\varepsilon>0$ such that when $W_{m}=2 C+\varepsilon$ IID results in higher welfare in area IV.

\section{Proof: See Appendix.}

No global statement can be made about the desirability of IID compared to the current regime, even when conditions in Proposition 6 hold and ambiguities concerning welfare effects in individual areas 
(I-IX) are eliminated. The reason is that aggregate welfare effects are given by a weighted average of the effects in individual areas. However, one may still speculate about the likely effects of switching to an IID regime by asking how industries' properties affect weights and magnitudes in areas I-IX. More precisely,

Proposition 7: If $W_{d}$ is large enough, then IID dominates the current regime.

Proof: See Appendix.

One immediate implication of Proposition 7 is that the magnitude of deadweight loss affects the desirability of IID. If increased competition eliminates large deadweight losses in the product market, IID improves welfare.

\section{D IS C USSION, EXTENSIONS, AND C O N CLUSION}

In this section, I consider several extensions and identify a few implications that follow from the model. The final subsection concludes.

\section{A. Comparison of Results under Incomplete Information and Complete Information}

Comparing results in Sections II and III reveals two important differences between results obtained under complete and incomplete information.

First, under complete information, it is relatively easy to identify when IID is beneficial. When our estimate of inventors' probability of success is sufficiently high (that is, $p \geq p$ ), IID is desirable. This conclusion cannot be reached under incomplete information, because firms' expectations concerning their and their rivals' likelihood of inventing the product are as important as their realized probabilities of success. Thus under incomplete information it is not possible to determine the desirability of IID by focusing on simple conditions based on firms' estimated probabilities of success.

Second, under complete information, the magnitude of deadweight losses eliminated by competition in the product market (that is, $\left.W_{d}-W_{m}\right)$ plays no role in determining whether IID is beneficial. Since probabilities of success are observable, we can determine conditions based on these probabilities under which IID is beneficial. Note that none of these conditions depends on $W_{d}{ }^{16}$ Under incomplete information, on the other hand, the size of $W_{d}$ is a major determinant

\footnotetext{
${ }^{16}$ See Section II.C to verify this claim.
} 
in whether IID is beneficial. ${ }^{17}$ This follows from the fact that we do not know what incentives IID will provide to a given firm, because its probability of success is unobservable. We only have beliefs concerning its probability of success. On the basis of these beliefs, we can only estimate how often firms will produce the product simultaneously under IID and generate welfare gains by increasing competition in the product market. Because these benefits are increasing in $W_{d \prime}$ they will offset costs generated by IID's effects of decreasing incentives to invent only if $W_{d}$ is sufficiently large. ${ }^{18}$

According to this comparison, determining conditions for the desirability of IID is harder under incomplete information. In particular, firms' beliefs about their and their rivals' probabilities of inventing the product and the size of eliminated deadweight losses through the application of IID (that is, $W_{d}-W_{m}$ ) become important considerations.

\section{B. Subsidies and Rewards}

It is interesting to consider the effects of complementing IID with small subsidies or awards. Recall that the main costs of IID involve decreased incentives to produce (that is, $p^{D}>p^{N}$ ). This disincentive can be cured by subsidizing firms that engage in research. Furthermore, such subsidies have no direct effect on IID's benefits, which come from increased competition in the ex post market for the invention. Therefore, subsidies could, in theory, cure the defects of IID and leave its benefits untouched. One could potentially design an IID-plus-subsidies regime that would dominate the current regime. One would, however, have to address two main problems associated with subsidies. First, errors in the determination of appropriate subsidies could be costly. If they are set too high, they will overincentivize firms. If they are set too low, they will be insufficient to offset the undesirable effects of IID. Second, subsidies would require an increase in aggregate taxes, which would lead to deadweight losses. It is not clear whether welfare gains from switching to an IID-plussubsidies regime would offset these losses.

${ }^{17}$ See Proposition 7.

${ }^{18}$ What "sufficiently large" means can be calculated by evaluating welfare under IID as a function of $W_{d^{\prime}}$ and evaluating welfare under the current regime. Since welfare under IID is increasing in $W_{d}$, one can find $W_{d}^{*}$, the critical $W_{d}$ that equalizes welfare under both regimes. Any $W_{d}$ greater than $W_{d}^{*}$ would be sufficiently large. 


\section{Independence of $\boldsymbol{p}_{i}$ and $f\left(p_{i}\right)$}

The instant model assumes that each firm draws its probability of success from a known distribution $f($.$) . A firm knows the likelihood$ with which it will get a good or a bad draw. Once it makes its draw, it attributes its particular probability of success to exogenous factors associated with the firm's own and unique properties. Accordingly, after it draws its probability of success $\left(p_{i}\right)$, it does not reevaluate its beliefs concerning the distribution from which its opponent will make its draw $\left(f\left(p_{j}\right)\right)$; that is, firm i's estimate of $f\left(p_{i}\right)$ is independent of $p_{i}$.

The reasonableness of this assumption is an empirical question whose answer depends on the product to be invented. For that reason, an extension analyzing the effects of relaxing this assumption may prove useful. In particular, $f($.$) , which represents the identical$ and independent distributions of $p_{i}$ and $p_{i^{\prime}}$ can be replaced with a joint probability distribution to capture correlations between a firm's own draw and its expectations concerning its rival's draw.

\section{Endogenous Determination of the Number of Players}

This paper focuses on the case where two rivals decide whether or not to invest in research. Future extensions can allow the number of players to be endogenously determined, by considering a larger, two-period game. In period one, a number of firms decide whether or not to incur an initial investment cost of $I$. In period two, $n$ firms who incurred the investment cost of $I$ play the $n$ player version of the game described in Section III. In this setting, firms would stop joining the second stage of the game when they estimate negative expected payoffs from doing so. That is, $n$ would reflect the satiation point of the research market for a particular invention. This extension would capture not only IID's effect on a fixed number of firms' incentives to conduct research, but also its effect on altering the number of firms interested in $R \& D$ in the first place. The latter effect will link welfare to changes in aggregate research costs and the aggregate probability of invention.

\section{E. Conclusion}

I have shown that IID's effects on welfare are ambiguous under incomplete information. I then identified particular conditions under which IID is likely to perform better than the current regime, and pointed out that these conditions are more complex than they would be in a complete information setting. Verifying whether these 
conditions are met in reality requires access to information that would be extremely hard to find and quantify, including information concerning R\&D firms' expectations regarding their and their rivals' likelihood of inventing a product, the social value of the product sought to be invented, deadweight losses arising from the noncompetitive market structure for a patented product, research costs, and private benefits to successful firms under both the current regime and IID. It therefore seems unlikely that one can accurately determine whether switching to an IID regime would increase welfare.

\section{A P P E N D I X}

Proof of Proposition 2: (i) Let $Z=\left\{q \in[0,1]\right.$ such that $\left.s_{j}(q)=R\right\}$ and let $\underline{Z}=[0,1] \backslash Z$.

Then, given any strategy $s_{i}$, firm i's expected utility from conducting research is given by:

$$
V_{i}\left(s_{i}, p_{i}\right)=\int_{p j \in Z} u_{i}\left(R, R_{i} p_{i}\right) f\left(p_{j}\right) d p_{j}+\int_{p j \in \underline{Z}} u_{i}\left(\underline{R}, R_{i} p_{i}\right) f\left(p_{i}\right) d p_{j}
$$

plugging in the corresponding values for $\mathrm{u}_{i}$ as defined in (10), we have:

$$
V_{i}\left(s_{j}, p_{i}\right)=\int_{p_{i} \in Z}\left[p_{i}\left(1-p_{i}\right) \pi_{m}+p_{i} p_{i} \rho\right] f\left(p_{i}\right) d p_{j}+\int_{p_{j} \in Z} p_{i} \pi_{m} f\left(p_{i}\right) d p_{j}
$$

It follows that in (A.2) both integrands and therefore $V_{i}$ are increasing in $p_{i^{*}}$

(ii) Follows trivially from part (i). Q.E.D.

Proof of Proposition 3: Proposition 2 establishes that any equilibrium must be of the form $S=\left(s_{p}{ }^{c}, s_{q}{ }^{c}\right)$. Symmetry requires that $p^{C}=q^{C}$, in which case $S$ is denoted as $S_{p}^{c}$. A symmetric strategy profile $S_{p}^{c}$ is an equilibrium profile iff

$$
V_{i}\left(s_{p^{c}}, p_{i}\right) \geq \int_{0}^{1} u_{i}\left(s_{p^{c}}\left(p_{j}\right), \underline{R}_{i} p_{i}\right) f\left(p_{j}\right) d p_{j}=0
$$

for all $p_{i} \geq p^{c}$ and

$$
V_{i}\left(s_{p^{c}}, p_{i}\right)<\int_{0}^{1} u_{i}\left(s_{p^{c}}\left(p_{i}\right), \underline{R}_{i} p_{i}\right) f\left(p_{i}\right) d p_{j}=0
$$

for all $p_{i}<p^{c}$, where $V_{i}$ is firm i's expected utility from conducting research.

This follows, because (i) (A.3) and (A.4) imply that $s_{p}{ }^{c}$ is a best response to $S_{p}^{c}$ and (ii) whenever firms' strategies are best responses to each other we have an equilibrium. 
But part (i) of proposition 2 implies that (A.3) and (A.4) hold iff $V_{i}\left(s_{p}^{c}, p^{c}\right)=0$, which is equivalent to

$$
\int_{0}^{p^{c}} p_{c} \pi_{m} f\left(p_{j}\right) d p_{j}+\int_{p^{c}}^{1}\left[p_{c}\left(1-p_{j}\right) \pi_{m}+p^{c} p_{i} \rho\right] f\left(p_{j}\right) d p_{j}=C
$$

To see this, note that $Z$ as referred to in (A.2) is equal to $\left[p^{C}, 1\right]$ when $s_{i}=s_{p}{ }^{c}$, and replace $p_{i}$ in (A.2) with $p^{c}$. To demonstrate existence and uniqueness it will be convenient to define the left-hand side of (A.5) as a function of $p^{c}$. Accordingly, let

$$
g\left(p^{c}\right)=\int_{0}^{p^{c}} p^{c} \pi_{m} f\left(p_{i}\right) d p_{j}+\int_{p^{c}}^{1}\left[p^{c}\left(1-p_{i}\right) \pi_{m}+p^{c} p_{i} \rho\right] f\left(p_{i}\right) d p_{j}
$$

Differentiating $g$ with respect to $p^{C}$ establishes the fact that $g$ is increasing in $p^{C}$ :

$$
\begin{aligned}
g_{p} & =\pi_{m} F\left(p^{c}\right)+\left(p^{c}\right)^{2} f\left(p^{c}\right)\left(\pi_{m}-\rho\right) \\
& +\int_{p^{c}}^{1}\left[\left(1-p_{i}\right) \pi_{m}+p_{i} \rho\right] f\left(p_{i}\right) d p_{j}>0
\end{aligned}
$$

where $F($.$) is the cumulative distribution function associated with$ $f($.$) . To see how the inequality follows, note that the first and third$ terms are obviously positive. The second term is also positive since $\rho<\pi_{m}$.

Next, note that

$$
g(0)=0<C \text { and } g(1)=\pi_{m}>C
$$

Hence, the intermediate value theorem applied to facts captured by (A.7) and (A.8) imply that there exists a unique and interior value of $p^{C}$ that satisfies (A.5). Q.E.D.

Proof of Proposition 4: (A.5) identifies the condition that $p^{C}$ must satisfy under both regimes. The value of $p^{C}$ that satisfies (A.5) depends on the legal regime only through the regime's effect on $\rho$. Hence, by applying the implicit function theorem, the effects of an increase in $\rho$ on $p^{C}$ can be determined:

$$
\begin{aligned}
\frac{\partial p^{c}}{\partial \rho} & =-\frac{9 \rho}{9 p^{c}} \\
& =\frac{p^{c} \int_{p^{c}}^{1} p_{i} f\left(p_{j}\right) d p_{j}}{\pi_{m} F\left(p^{c}\right)+\left(p^{c}\right)^{2} f\left(p^{c}\right)\left(\pi_{m}-\rho\right)+\int_{p^{c}}^{1}\left[\left(1-p_{j}\right) \pi_{m}+p_{i} \rho\right] f\left(p_{j}\right) d p_{j}}<0
\end{aligned}
$$


Next, recall that

$$
\rho(D)=\pi_{d}<\frac{\pi_{m}}{2}=\rho(N)
$$

Hence,

$$
p^{D}>p^{N}
$$

Q.E.D.

Proof of Proposition 5: The proof of parts (i) and (iii) follow immediately from the explanations provided in Section III following the statement of Proposition 5.

(ii) Let $p_{m}=\max \left\{p_{1}, p_{2}\right\}$. At any point $\left(p_{1}, p_{2}\right)$ that falls in areas VII or VIII, expected social welfare is 0 under IID and is given by $p_{m} W_{m}-C$ under the current regime. But in these areas, it follows that $p_{m} \pi_{m}>C$, since firm $m$ would not engage in research otherwise. Hence, $p_{m} W_{m}-C>p_{m} \pi_{m}-C>0$, which implies that social welfare is higher under the current regime at any point that falls in areas VII or VIII.

(iv) Area IV: At any point $\left(p_{1}, p_{2}\right)$ that falls in area IV, expected social welfare is 0 under an IID regime and $\left[1-\left(1-p_{1}\right)\left(1-p_{2}\right)\right] W_{m}-$ $2 \mathrm{C}$ under the current regime. Hence, an IID regime results in higher welfare iff

$$
\left[1-\left(1-p_{1}\right)\left(1-p_{2}\right)\right] W_{m}<2 C
$$

Next, note that $1-\left(1-p_{1}\right)\left(1-p_{2}\right)<1-\left(1-p^{D}\right)^{2}=p^{D}\left(2-p^{D}\right)$ for all $\left(p_{1}\right.$, $\left.p_{2}\right)$ in area IV. Furthermore, $p^{D}\left(2-p^{D}\right)$ is increasing in $p^{D}$ for all $p^{D}$ $\in[0,1)$. Accordingly, the left-hand side of (A.12) attains the highest possible value when $p_{1}=p_{2}=p^{D}$ and $p^{D}$ is as high as possible. But from the proof of Proposition 3 we know that $p^{D}<1$. Hence, the highest value the left-hand side of (A.12) can take is lower than $W_{m}$. Therefore, it follows that there exists $\varepsilon>0$ such that $W_{m}=2 C$ $+\varepsilon$ implies that $(A .12)$ holds for all $\left(p_{1}, p_{2}\right)$ in area IV. Finally, note that $W_{m}$ does not have an upper bound, and if it is sufficiently high, (A.12) does not hold for any $\left(p_{1}, p_{2}\right)$ in area IV. Accordingly, welfare effects are ambiguous in area $I V$.

Areas II and III: At any point $\left(p_{1}, p_{2}\right)$ that falls in area II, expected social welfare is $p_{2} W_{m}-C$ under an IID regime and $\left[1-\left(1-p_{1}\right)\right.$ $\left.\left(1-p_{2}\right)\right] W_{m}-2 C$ under the current regime. Hence, an IID regime results in higher welfare iff

$$
C>p_{1}\left(1-p_{2}\right) W_{m}
$$


Next, note that $p^{D}\left(1-p^{D}\right)>p_{1}\left(1-p_{2}\right)$ for all $\left(p_{1}, p_{2}\right)$ in area II. Hence, IID is more desirable at any point in area II if

$$
C>p^{D}\left(1-p^{D}\right) W_{m}
$$

But the maximum value of $p^{D}\left(1-p^{D}\right)$ is 0.25. Accordingly, if $W_{m}$ $<4 C$, it follows that IID leads to higher welfare in the entirety of area II. Finally, note that $W_{m}$ does not have an upper bound, and if it is sufficiently high IID will be inferior in all points in this area. Hence, IID's welfare effects in this area are ambiguous.

The same conclusion holds for area III, because of the symmetry between areas II and III. Q.E.D.

Proof of Proposition 6: Both parts are proven in the proof of Proposition 4 part (iv).

Proof of Proposition 7: The claim follows from the fact that $W_{d}$ affects only the gains from IID in area I, and does not affect firms' incentives. Hence, given any economy, there is a large enough $W_{d}$, such that social welfare is greater under IID than under the current regime. Q.E.D. 